\title{
Efficient CAN catalyzed synthesis of $1 H$-indazolo[1,2-b] phthalazine-1,6,11-triones: An eco-friendly protocol
}

\author{
MAZAAHIR Kidwai*, RITIKA Chauhan \& ANWAR Jahan \\ Green Chemistry Research Laboratory, Department of Chemistry, University of Delhi, Delhi-110007, India
}

Received August 12, 2011; accepted October 8, 2011; published online March 23, 2012

\begin{abstract}
A convenient, economical and green approach to the synthesis of $1 H$-indazolo[1,2- $b$ ]phthalazine-1,6,11-trione derivatives has been achieved via a one-pot protocol using phthalhydrazide, a cyclic- $\beta$-diketone and an aldehyde in the presence of a ceric ammonium nitrate catalyst in polyethylene glycol. The simple work up, mild conditions, excellent yields, inexpensive and non-toxic catalyst and simple solvent recyclability render this protocol both attractive and economically viable.
\end{abstract}

ceric ammonium nitrate, polyethylene glycol, $1 H$-indazolo[1,2-b]phthalazine-1,6,11-triones, recyclability, green chemistry

Citation: $\quad$ Mazaahir K, Ritika C, Anwar J. Efficient CAN catalyzed synthesis of $1 H$-indazolo[1,2-b]phthalazine-1,6,11-triones: An eco-friendly protocol. Chin Sci Bull, 2012, 57: 2273-2279, doi: 10.1007/s11434-012-5081-7

Multi-component reactions (MCRs) offer a number of fascinating and challenging transformations in organic synthesis [1-7]. Atom-economy, convergent character, operational simplicity, structural diversity, and complexity are the major advantages associated with multi-component reactions. Furthermore, multi-component reactions are emerging as a powerful tool for the synthesis of biologically important compounds [8,9]. In drug discovery, as well as "Green Chemistry", MCRs are the techniques of choice for the high-throughput synthesis of compounds from a cost- and time point of view $[10,11]$.

Over the past few decades, a tremendous upsurge of interest has been observed in the synthesis of nitrogencontaining heterocyclic compounds, owing to their abundance in nature and essentiality to life. Their applications to biologically active pharmaceuticals, agrochemicals, and functional materials are also becoming increasingly important [12-16]. Phthalazine derivatives, that comprise two bridgehead nitrogen atoms in a fused ring system, have attracted considerable attention because of their pharmacological, and biological properties, and potential clinical application [17-34]. These compounds have also proved to be

*Corresponding author (email: kidwai.chemistry@gmail.com) promising luminescence materials and fluorescence probes [35]. Several methods have been reported for the synthesis of phthalazine derivatives [36-41]. Unfortunately, many of these methods are plagued with a number of limitations, such as harsh reaction conditions, unsatisfactory yields, tedious work-up procedures, relatively long reaction times, poor solvent scope and the use of stoichiometric and relatively expensive reagents. Therefore, improved methods, in terms of operational simplicity, reusability and economic viability are highly desirable.

Recently, ceric ammonium nitrate (CAN) has been utilized for various organic transformations [42-45]. The most characteristic feature of CAN is that it can act as a watercompatible Lewis acid in aqueous solvents. Additionally, advantages such as low-cost, eco-friendly nature, ease of handling, non-toxic nature make CAN an attractive catalyst for a variety of synthetic transformations. Regulatory pressures are increasingly focusing on the use, manufacture and disposal of organic solvents, and thus, the development of non-hazardous alternatives (one of the several goals for green chemistry and engineering) is vitally important for the continued and sustainable development of the chemical processes. Recently, polyethylene glycol (PEG) and solutions thereof have been introduced as potential green 
solvent systems [46-48]. They have replaced a number of other "neoteric solvents", such as ionic liquids, super critical carbon dioxide, and micellar systems, whose toxicological properties, short and long-term hazardous nature, and biodegradability are not completely understood. Their low cost, reduced flammability, reduced toxicity, recyclability, non-halogenated composition, easy degradability, and miscibility with wide variety of organic solvents are just some of the key features that render PEG an attractive alternative solvent in organic synthesis [49-53]. Keeping within our theme of green chemistry and in a continuation of our studies for the development of cheap and environmentally benign methodologies for organic synthesis [54-56], we decided to examine the versatility of CAN as a catalyst and PEG 400 as solvent for the synthesis of $1 H$ indazolo[1,2-b]phthalazine-1,6,11-triones.

\section{Experimental}

\subsection{Materials and methods}

All chemicals were used as received from Sigma-Aldrich without any further purification. All reactions were monitored by thin-layer chromatography (TLC), using aluminium plates coated with silica gel $\mathrm{F}_{254}$; (Merck) using ethyl acetate, and hexane (40/60) as eluent. Spots were visualized using either UV light or an iodine chamber. Melting points were determined in using a Thomas Hoover melting point apparatus (uncorrected). IR spectra were recorded on a Perkin-Elmer FTIR-1710 spectrophotometer, using $\mathrm{KBr}$ pellets/-nujol film. ${ }^{1} \mathrm{H}$ and ${ }^{13} \mathrm{C}$ NMR spectra were recorded on a JEOL JNM-ECX 400P FT NMR spectrometer with TMS as the internal standard. Chemical shifts are on a $\delta$ scale and coupling constant values $(J)$ are in Hertz $(\mathrm{Hz})$. Elemental analyses were performed on a Hereaus CHN rapid analyzer. The temperature of the reaction mixture was measured using a non-contact infrared mini gun thermometer (AZ minigun type, model 8868).

\subsection{General procedure for the synthesis of $1 \mathrm{H}$ -} indazolo[1,2-b]phthalazine-1,6,11-trione derivatives

To a $50 \mathrm{~mL}$ round-bottom flask, phthalhydrazide $\mathbf{1}$, aldehyde (RCHO) 2a-2q, cyclic $\beta$-diketone 3a $\left(\mathrm{C}_{6} \mathrm{H}_{8} \mathrm{O}_{2}\right), \mathbf{3 b}$ $\left(\mathrm{C}_{8} \mathrm{H}_{12} \mathrm{O}_{2}\right)$ and PEG $400(2 \mathrm{~mL})$ were added. CAN $(5 \mathrm{~mol} \%)$ was then added and reaction mixture was stirred at $50^{\circ} \mathrm{C}$ for the appropriate time, as summarized in Table 1. The progress of the reaction was monitored by TLC. Upon completion of the reaction, the mixture was cooled in a dry ice-acetone bath, to precipitate the PEG 400, and extracted with ether (PEG is insoluble in ether). The ether layer was decanted, dried over anhydrous $\mathrm{Na}_{2} \mathrm{SO}_{4}$ and concentrated under reduced pressure to afford the desired product. The crude product was purified by column chromatography on silica gel (100-200 mesh size), using hexane/ethyl acetate as eluent. This provided the pure respective $1 \mathrm{H}$ indazolo[1,2-b]phthalazine-1,6,11-trione derivative, $(\mathbf{4 a}-\mathbf{4 s})$. The recovered PEG 400 phase was reused for consecutive reactions. All products were characterized on the basis of their spectral analysis (IR, ${ }^{1} \mathrm{H}$ NMR, ${ }^{13} \mathrm{C}$ NMR and elemental analysis) and melting point determination [37,57-60].

Table 1 CAN catalyzed synthesis of $1 H$-indazolo[1,2-b]phthalazine-1,6,11-triones ${ }^{\text {a) }}$

\begin{tabular}{|c|c|c|c|c|c|}
\hline Entry & $\mathrm{R}$ & $\mathrm{R}^{\prime}$ & Product & Time (h) & Yield $(\%)^{\mathrm{b}}$ \\
\hline 1 & $\mathrm{Ph}(\mathbf{2 a})$ & $\mathrm{H}(\mathbf{3 a})$ & $4 a$ & 2 & 94 \\
\hline 2 & $4-\mathrm{Br}-\mathrm{C}_{6} \mathrm{H}_{4}(\mathbf{2 b})$ & $\mathrm{H}$ & $4 b$ & 2.5 & 92 \\
\hline 3 & $3-\mathrm{NO}_{2}-\mathrm{C}_{6} \mathrm{H}_{4}(\mathbf{2 c})$ & $\mathrm{H}$ & $4 c$ & 3.5 & 94 \\
\hline 4 & $4-\mathrm{Me}-\mathrm{C}_{6} \mathrm{H}_{4}(\mathbf{2 d})$ & $\mathrm{H}$ & $4 d$ & 2 & 94 \\
\hline 5 & $3-\mathrm{HO}-\mathrm{C}_{6} \mathrm{H}_{4}(\mathbf{2 e})$ & $\mathrm{H}$ & $4 e$ & 2 & 92 \\
\hline 6 & 4-HO-3-MeO- $\mathrm{C}_{6} \mathrm{H}_{3}(\mathbf{2 f})$ & $\mathrm{H}$ & $4 f$ & 3 & 94 \\
\hline 7 & $4-\mathrm{Me}_{2} \mathrm{~N}-\mathrm{C}_{6} \mathrm{H}_{4}(\mathbf{2 g})$ & $\mathrm{H}$ & $4 \mathrm{~g}$ & 2.5 & 90 \\
\hline 8 & $\mathrm{Ph}$ & $\operatorname{Me}(\mathbf{3 b})$ & $4 \mathrm{~h}$ & 2 & 94 \\
\hline 9 & $4-\mathrm{Cl}-\mathrm{C}_{6} \mathrm{H}_{4}(\mathbf{2 h})$ & $\mathrm{Me}$ & $4 i$ & 2 & 90 \\
\hline 10 & $4-\mathrm{MeO}-\mathrm{C}_{6} \mathrm{H}_{4}(\mathbf{2 i})$ & $\mathrm{Me}$ & $4 j$ & 2 & 92 \\
\hline 11 & $4-\mathrm{NO}_{2}-\mathrm{C}_{6} \mathrm{H}_{4}(\mathbf{2} \mathbf{j})$ & $\mathrm{Me}$ & $4 k$ & 3.5 & 90 \\
\hline 12 & 4-Me- $\mathrm{C}_{6} \mathrm{H}_{4}$ & $\mathrm{Me}$ & 41 & 2.5 & 94 \\
\hline 13 & $2-\mathrm{HO}-\mathrm{C}_{6} \mathrm{H}_{4}(\mathbf{2 k})$ & $\mathrm{Me}$ & $4 m$ & 2 & 90 \\
\hline 14 & Piperonyl (2I) & $\mathrm{Me}$ & $4 n$ & 3 & 88 \\
\hline 15 & 2-HO-1-Naphthyl (2m) & $\mathrm{Me}$ & 40 & 3.5 & 89 \\
\hline 16 & Ethyl (2n) & $\mathrm{H}$ & $4 p$ & 4 & 62 \\
\hline 17 & Propyl (2o) & $\mathrm{H}$ & $4 q$ & 4 & 64 \\
\hline 18 & Isobutyl (2p) & $\mathrm{Me}$ & $4 r$ & 4 & 62 \\
\hline 19 & Hexyl (2q) & $\mathrm{Me}$ & $4 s$ & 4 & 63 \\
\hline
\end{tabular}

a) Reaction conditions: phthalhydrazide $1(1 \mathrm{mmol})$, aldehyde $\mathbf{2 a - 2 q}(1 \mathrm{mmol})$, cyclic $\beta$-diketone 3a, 3b $(1 \mathrm{mmol})$; catalyst: CAN $(5 \mathrm{~mol} \%) ; T: 50^{\circ} \mathrm{C}$; solvent: PEG 400 (2 mL). b) Isolated yields. 


\subsection{Spectral data for the synthesized compounds}

(1) 2,3,4,13-Tetrahydro-13-phenyl-1 $H$-indazolo[1,2- $b]$ phthalazine-1,6,11-trione (4a). Yellow solid, mp. $222-225^{\circ} \mathrm{C}$ [58]. IR (KBr, $\left.v_{\max } \mathrm{cm}^{-1}\right): 2920,1655,1630,1360,1310$, 1284, 754. ${ }^{1} \mathrm{H}$ NMR $\left(\mathrm{CDCl}_{3}, 400 \mathrm{MHz}, \mathrm{TMS}\right): \delta 8.24-8.35$ (m, 2H), 7.80-7.88 (m, 2H), 7.17-7.38 (m, 5H, Ar-H), 6.42 $(\mathrm{s}, 1 \mathrm{H}, \mathrm{Ar}-\mathrm{CH}), 3.26-3.63(\mathrm{~m}, 2 \mathrm{H}), 2.41-2.64(\mathrm{~m}, 2 \mathrm{H})$, 2.21-2.36 (m, 2H); ${ }^{13} \mathrm{C} \mathrm{NMR}\left(\mathrm{CDCl}_{3}, 100 \mathrm{MHz}\right): \delta 197.1$, $164.2,157.3,150.8,145.5,133.9,129.4,128.7,127.8$, 127.3, 126.5, 119.4, 65.4, 36.3, 26.8, 21.4. Anal. Calcd. for $\mathrm{C}_{21} \mathrm{H}_{16} \mathrm{~N}_{2} \mathrm{O}_{3}(\%)$ : C, 73.24; H, 4.68; N, 8.13; Found (\%): C, $73.11 ; \mathrm{H}, 4.60 ; \mathrm{N}, 8.05$.

(2) 2,3,4,13-Tetrahydro-13-(4-bromo-phenyl)- $1 H$-indazolo[1,2- $b]$ phthalazine-1,6,11-trione $(\mathbf{4 b})$. Yellow solid, mp. 279-282 ${ }^{\circ} \mathrm{C}$ [60]. IR (nujol, $v_{\max } \mathrm{cm}^{-1}$ ): 2926, 1656, 1621, 1368, 1309, 1284, 755, 668. ${ }^{1} \mathrm{H}$ NMR $\left(\mathrm{CDCl}_{3}, 400\right.$ $\mathrm{MHz}, \mathrm{TMS}): \delta 8.23-8.37(\mathrm{~m}, 2 \mathrm{H}), 7.82-7.88(\mathrm{~m}, 2 \mathrm{H})$, 7.15-7.46 (m, 4H, Ar-H), 6.43 (s, 1H, Ar-CH), 3.43-3.66 $(\mathrm{m}, 2 \mathrm{H}), 2.44-2.67(\mathrm{~m}, 2 \mathrm{H}), 2.23-2.37(\mathrm{~m}, 2 \mathrm{H}) ;{ }^{13} \mathrm{C}$ NMR $\left(\mathrm{CDCl}_{3}, 100 \mathrm{MHz}\right): \delta 194.4,155.6,154.2,153.2,135.3$, 134.5, 132.8, 132.6, 129.4, 128.3, 127.9, 123.7, 120.3, 51.7, 40.0, 36.5, 29.2. Anal. Calcd. for $\mathrm{C}_{21} \mathrm{H}_{15} \mathrm{BrN}_{2} \mathrm{O}_{3}(\%)$ : $\mathrm{C}$, 59.59; H, 3.57; N, 6.62; Found (\%): C, 59.50; H, 3.52; $\mathrm{N}, 6.51$.

(3) 2,3,4,13-Tetrahydro-13-(3-nitro-phenyl)-1H-indazolo[1,2-b]phthalazine-1,6,11-trione (4c). Light yellow solid, mp. $228-232^{\circ} \mathrm{C}$ [59]. IR (KBr, $\left.v_{\max } \mathrm{cm}^{-1}\right): 2923,1667,1624$, 1371, 1307, 1284, 958, 703. ${ }^{1} \mathrm{H}$ NMR $\left(\mathrm{CDCl}_{3}, 400 \mathrm{MHz}\right.$, TMS): $\delta 8.24-8.34(\mathrm{~m}, 2 \mathrm{H}), 7.81-7.87(\mathrm{~m}, 2 \mathrm{H}), 7.29-7.45$ (m, 4H, Ar-H), 6.38 (s, 1H, Ar-CH), 3.46-3.64 (m, 2H), 2.52-2.67 (m, 2H), 2.20-2.47 (m, 2H); ${ }^{13} \mathrm{C} \mathrm{NMR}\left(\mathrm{CDCl}_{3}\right.$, $100 \mathrm{MHz}): \delta 196.2,165.5,156.0,154.8,153.4,145.6,135.2$, $134.8,129.4,128.6,127.3,124.5,120.4,116.4$, 64.2, 36.8, 26.9, 21.3. Anal. Calcd. for $\mathrm{C}_{21} \mathrm{H}_{15} \mathrm{~N}_{3} \mathrm{O}_{5}(\%)$ : C, 64.78; $\mathrm{H}$, 3.88 ; N, 10.79; Found (\%): C, 64.65; H, 3.76; N, 10.65.

(4) 2,3,4,13-Tetrahydro-13-(4-methyl-phenyl)- $1 H$-indazolo[1,2- $b]$ phthalazine-1,6,11-trione (4d). Yellow solid, mp. 244-246 ${ }^{\circ} \mathrm{C}$ [59]. IR $\left(\mathrm{KBr}, v_{\max } \mathrm{cm}^{-1}\right): 2954,1657,1621$, $1365,1308,1265,957,704 .{ }^{1} \mathrm{H}$ NMR $\left(\mathrm{CDCl}_{3}, 400 \mathrm{MHz}\right.$, TMS): $\delta 8.24-8.36(\mathrm{~m}, 2 \mathrm{H}), 7.81-7.86(\mathrm{~m}, 2 \mathrm{H}), 7.11-7.34$ (m, 4H, Ar-H), 6.39 (s, 1H, Ar-CH), 3.52-3.61 (m, 2H), 2.43-2.62 (m, 2H), 2.29 (s, 3H, $\mathrm{CH}_{3}$ ), 2.00-2.07 (m, 2H); ${ }^{13} \mathrm{C} \mathrm{NMR}\left(\mathrm{CDCl}_{3}, 100 \mathrm{MHz}\right): \delta 196.0,193.2,164.2,156.4$, $142.4,136.4,133.9,133.4,129.7,129.3,127.7,127.5$, 120.4, 116.9, 65.5, 37.6, 29.4, 21.6, 21.0. Anal. Calcd. for $\mathrm{C}_{22} \mathrm{H}_{18} \mathrm{~N}_{2} \mathrm{O}_{3}(\%): \mathrm{C}, 73.73 ; \mathrm{H}, 5.06 ; \mathrm{N}, 7.82$; Found (\%): C, $73.65 ; \mathrm{H}, 4.89 ; \mathrm{N}, 7.65$.

(5) 2,3,4,13-Tetrahydro-13-(3-hydroxy-phenyl)- $1 \mathrm{H}$-indazolo[1,2-b]phthalazine-1,6,11-trione (4e). White solid, mp. 266-268 ${ }^{\circ} \mathrm{C}$ [60]; IR (nujol, $v_{\max } \mathrm{cm}^{-1}$ ): 3404, 2853, 1640, $1618,1378,1311,1214,771,674 .{ }^{1} \mathrm{H}$ NMR $\left(\mathrm{CDCl}_{3}, 400\right.$ MHz, TMS): $\delta$ 8.24-8.38 (m, 2H), 7.83-7.89 (m, 2H), 6.73-7.06 (m, 4H, Ar-H), 6.43 (s, 1H, Ar-CH), 4.76 (br s, $1 \mathrm{H}, \mathrm{OH}), 3.52-3.62(\mathrm{~m}, 2 \mathrm{H}), 2.53-2.68(\mathrm{~m}, 2 \mathrm{H}), 2.22-2.47$ $(\mathrm{m}, 2 \mathrm{H}) ;{ }^{13} \mathrm{C} \mathrm{NMR}\left(\mathrm{CDCl}_{3}, 100 \mathrm{MHz}\right): \delta 197.3,165.2$, $163.0,158.8,156.2,139.3,138.4,133.7,132.7,131.8$, 131.5, 130.3, 129.3, 124.2, 119.3, 68.4, 37.5, 29.8, 25.7. Anal. Calcd. for $\mathrm{C}_{21} \mathrm{H}_{16} \mathrm{~N}_{2} \mathrm{O}_{4}(\%)$ : C, 69.99; H, 4.48; N, 7.77; Found (\%): C, 69.75; H, 4.38; N, 7.62.

(6) 2,3,4,13-Tetrahydro-13-(4-hydroxy-3-methoxy-phenyl)$1 H$-indazolo[1,2-b]phthalazine-1,6,11-trione (4f). Yellow solid, mp. 202-206 ${ }^{\circ}$ C. IR (nujol, $v_{\max } \mathrm{cm}^{-1}$ ): 3536, 2920, $1668,1596,1468,1364,1272,1215,1128,960,630 .{ }^{1} \mathrm{H}$ $\mathrm{NMR}\left(\mathrm{CDCl}_{3}, 400 \mathrm{MHz}, \mathrm{TMS}\right): \delta 8.25-8.36(\mathrm{~m}, 2 \mathrm{H})$, $7.82-7.88(\mathrm{~m}, 2 \mathrm{H}), 6.52-6.56(\mathrm{~m}, 3 \mathrm{H}, \mathrm{Ar}-\mathrm{H}), 6.38(\mathrm{~s}, 1 \mathrm{H}$, Ar-CH), 4.73 (br s, 1H, OH), 3.97 (s, 3H, $\left.\mathrm{OCH}_{3}\right), 2.51-2.64$ $(\mathrm{m}, 2 \mathrm{H}), 2.25-2.42(\mathrm{~m}, 2 \mathrm{H}), 1.96-2.07(\mathrm{~m}, 2 \mathrm{H}) ;{ }^{13} \mathrm{C} \mathrm{NMR}$ $\left(\mathrm{CDCl}_{3}, 100 \mathrm{MHz}\right): \delta$ 197.3, 164.3, 152.6, 146.4, 143.2, 137.6, 130.5, 129.5, 127.3, 120.7, 115.2, 112.1, 110.4, 64.9, 55.7, 37.8, 27.0, 22.8. Anal. Calcd. for $\mathrm{C}_{22} \mathrm{H}_{18} \mathrm{~N}_{2} \mathrm{O}_{5}(\%)$ : C, 67.69; H, 4.65; N, 7.18; Found (\%): C, 67.56; H, 4.58; N, 7.02 .

(7) 2,3,4,13-Tetrahydro-13-(4-dimethylamino-phenyl)- $1 \mathrm{H}$ indazolo[1,2-b]phthalazine-1,6,11-trione (4g). Red solid, mp. 255-256 ${ }^{\circ} \mathrm{C}$ [59]. IR (nujol, $v_{\max } \mathrm{cm}^{-1}$ ): 2924, 1672, 1624, 1365, 1313, 1226, 671. ${ }^{1} \mathrm{H}$ NMR $\left(\mathrm{CDCl}_{3}, 400 \mathrm{MHz}\right.$, TMS): $\delta 8.22-8.32(\mathrm{~m}, 2 \mathrm{H}), 7.79-7.83(\mathrm{~m}, 2 \mathrm{H}), 7.16(\mathrm{~d}, J=$ $9.0 \mathrm{~Hz}, 2 \mathrm{H}), 6.73$ (d, $J=8.8 \mathrm{~Hz}, 2 \mathrm{H}), 6.40$ (s, 1H, Ar-CH), 3.07 (s, 6H, N( $\left.\left.\mathrm{CH}_{3}\right)_{2}\right), 2.50-2.64(\mathrm{~m}, 2 \mathrm{H}), 2.25-2.37$ (m, $2 \mathrm{H}), 1.93-2.03(\mathrm{~m}, 2 \mathrm{H}) ;{ }^{13} \mathrm{C} \mathrm{NMR}\left(\mathrm{CDCl}_{3}, 100 \mathrm{MHz}\right): \delta$ $196.2,164.5,154.6,153.1,137.2,133.4,129.0,128.4,128.0$, 126.3, 118.3, 112.6, 65.3, 41.3, 40.7, 30.1, 21.8. Anal. Calcd. for $\mathrm{C}_{23} \mathrm{H}_{21} \mathrm{~N}_{3} \mathrm{O}_{3}(\%)$ : C, 71.30; H, 5.46; N, 10.85; Found (\%): C, 71.16; H, 5.33; N, 10.74 .

(8) 2,3,4,13-Tetrahydro-3,3-dimethyl-13-phenyl- $1 H$-indazolo[1,2-b]phthalazine-1,6,11-trione (4h). Light yellow solid, mp. 202-204 ${ }^{\circ} \mathrm{C}$ [37]. IR ( $\left.\mathrm{KBr}, v_{\max } \mathrm{cm}^{-1}\right)$ : 2959, 1662 , $1623,1361,1308,1262,791 .{ }^{1} \mathrm{H}$ NMR $\left(\mathrm{CDCl}_{3}, 400 \mathrm{MHz}\right.$, TMS): $\delta 8.25-8.35(\mathrm{~m}, 2 \mathrm{H}), 7.83-7.85(\mathrm{~m}, 2 \mathrm{H}), 7.20-7.41$ (m, 5H, Ar-H), 6.44 (s, 1H, Ar-CH), 3.20 and 3.42 (AB-q system, $J=19.04 \mathrm{~Hz}, 2 \mathrm{H}), 2.45(\mathrm{~s}, 2 \mathrm{H}), 1.08\left(\mathrm{~s}, 3 \mathrm{H}, \mathrm{CH}_{3}\right)$, $0.97\left(\mathrm{~s}, 3 \mathrm{H}, \mathrm{CH}_{3}\right) ;{ }^{13} \mathrm{C} \mathrm{NMR}\left(\mathrm{CDCl}_{3}, 100 \mathrm{MHz}\right): \delta 196.4$, $162.2,155.9,150.7,144.0,128.9,128.7,128.3,127.7$, 127.1, 126.3, 115.6, 64.9, 50.9, 40.8, 28.4, 27.3. Anal. Calcd. for $\mathrm{C}_{23} \mathrm{H}_{20} \mathrm{~N}_{2} \mathrm{O}_{3}(\%)$ : C, 74.18; H, 5.41; N, 7.52; Found (\%): C, 74.03; H, 5.29 ; N, 7.40.

(9) 2,3,4,13-Tetrahydro-3,3-dimethyl-13-(4-chloro-phenyl)- $1 H$-indazolo[1,2- $b]$ phthalazine-1,6,11-trione (4i). Light yellow solid, mp. $262-266^{\circ} \mathrm{C}$ [37]. IR $\left(\mathrm{KBr}, v_{\max } \mathrm{cm}^{-1}\right)$ : 2962, 1660, 1624, 1362, 1310, 1263, 791. ${ }^{1} \mathrm{H}$ NMR $\left(\mathrm{CDCl}_{3}\right.$, $400 \mathrm{MHz}, \mathrm{TMS}): \delta 8.25-8.36(\mathrm{~m}, 2 \mathrm{H}), 7.85-7.87(\mathrm{~m}, 2 \mathrm{H})$, 7.29-7.36 (m, 4H, Ar-H), $6.41(\mathrm{~s}, 1 \mathrm{H}, \mathrm{Ar}-\mathrm{CH}), 3.20$ and 3.42 (AB-q system, $J=19.04 \mathrm{~Hz}, 2 \mathrm{H}), 2.35$ (s, 2H), 1.20 (s, $\left.3 \mathrm{H}, \mathrm{CH}_{3}\right), 0.98\left(\mathrm{~s}, 3 \mathrm{H}, \mathrm{CH}_{3}\right) ;{ }^{13} \mathrm{C} \mathrm{NMR}\left(\mathrm{CDCl}_{3}, 100 \mathrm{MHz}\right): \delta$ 196.4, 162.4, 155.7, 151.0, 142.6, 134.6, 132.0, 129.7, 128.5, 128.2, 127.7, 115.2, 64.3, 50.8, 40.8, 29.3, 27.3. Anal. Calcd. for $\mathrm{C}_{23} \mathrm{H}_{19} \mathrm{ClN}_{2} \mathrm{O}_{3}(\%)$ : C, 67.90; H, 4.71; N, 6.89; Found (\%): C, 67.76; H, 4.62; N, 6.75.

(10) 2,3,4,13-Tetrahydro-3,3-dimethyl-13-(4-methoxy- 
phenyl)-1 $1 H$-indazolo[1,2-b]phthalazine-1,6,11-trione (4j). White solid, mp. 202-206 ${ }^{\circ} \mathrm{C}$ [58]. IR ( $\left.\mathrm{KBr}, v_{\max } \mathrm{cm}^{-1}\right)$ : 3015, 2898, 1664, 1495, 1377, 1304, 1262, 791. ${ }^{1} \mathrm{H}$ NMR $\left(\mathrm{CDCl}_{3}, 400 \mathrm{MHz}, \mathrm{TMS}\right): \delta 8.25-8.33$ (m, 2H), 7.80-7.86 (m, 2H), 6.71-7.18 (m, 4H, Ar-H), 6.39 (s, 1H, Ar-CH), $3.71\left(\mathrm{~s}, 3 \mathrm{H}, \mathrm{OCH}_{3}\right.$ ), 3.19 and 3.42 (AB-q system, $J=19.1$ $\mathrm{Hz}, 2 \mathrm{H}), 2.42$ (s, 2H), 1.07 (s, 3H, $\left.\mathrm{CH}_{3}\right), 0.97$ (s, 3H, $\mathrm{CH}_{3}$ ); ${ }^{13} \mathrm{C}$ NMR $\left(\mathrm{CDCl}_{3}, 100 \mathrm{MHz}\right): \delta 195.5,162.0,159.4,157.7$, 143.1, 136.2, 132.5, 129.3, 128.7, 127.6. 115.8, 113.4, 55.1, 50.7, 40.8, 32.2, 29.3, 27.3. Anal. Calcd. for $\mathrm{C}_{24} \mathrm{H}_{22} \mathrm{~N}_{2} \mathrm{O}_{4}$ (\%): C, 71.63; H, 5.51; N, 6.96; Found (\%): C, 71.51; H, $5.38 ; \mathrm{N}, 6.85$.

(11) 2,3,4,13-Tetrahydro-3,3-dimethyl-13-(4-nitro-phenyl)$1 H$-indazolo[1,2- $b]$ phthalazine-1,6,11-trione (4k). Creamy white solid, mp. 224-226 ${ }^{\circ} \mathrm{C}$ [58]. IR ( $\left.\mathrm{KBr}, v_{\max } \mathrm{cm}^{-1}\right): 3013$, $2959,1661,1593,1375,1345,1262,851 .{ }^{1} \mathrm{H}$ NMR $\left(\mathrm{CDCl}_{3}\right.$, $400 \mathrm{MHz}, \mathrm{TMS}): \delta 8.22-8.38(\mathrm{~m}, 2 \mathrm{H}), 7.86-7.89(\mathrm{~m}, 2 \mathrm{H})$, 7.23-7.62 (m, 4H, Ar-H), 6.51 (s, 1H, Ar-CH), 3.23 and 3.43 (AB-q system, $J=21.2 \mathrm{~Hz}, 2 \mathrm{H}$ ), 2.47 (s, 2H), 1.23 (s, $\left.3 \mathrm{H}, \mathrm{CH}_{3}\right), 1.11$ (s, $\left.3 \mathrm{H}, \mathrm{CH}_{3}\right) ;{ }^{13} \mathrm{C} \mathrm{NMR}\left(\mathrm{CDCl}_{3}, 100 \mathrm{MHz}\right)$ : $\delta 196.1,162.9,151.2,148.7,146.5,146.1,133.7,132.3$, 129.2, 127.6, 123.5, 114.9, 63.8, 50.4, 46.9, 46.3, 29.5, 27.4; Anal. Calcd. for $\mathrm{C}_{23} \mathrm{H}_{19} \mathrm{~N}_{3} \mathrm{O}_{5}(\%)$ : C, 66.18; H, 4.59; N, 10.07; Found (\%): C, 66.08; H, 4.44; N, 10.02.

(12) 2,3,4,13-Tetrahydro-3,3-dimethyl-13-(4-methyl-phenyl)-1 $H$-indazolo[1,2-b]phthalazine-1,6,11-trione (4I). Yellow solid, mp. $226-228^{\circ} \mathrm{C}$ [37]. IR $\left(\mathrm{KBr}, v_{\max } \mathrm{cm}^{-1}\right)$ : 3017, 2960, 2898, 1664, 1492, 1350, 1263, 791. ${ }^{1} \mathrm{H}$ NMR $\left(\mathrm{CDCl}_{3}\right.$, $400 \mathrm{MHz}, \mathrm{TMS}): \delta 8.23-8.33(\mathrm{~m}, 2 \mathrm{H}), 7.80-7.86(\mathrm{~m}, 2 \mathrm{H})$, 6.98-7.16 (m, 4H, Ar-H), 6.39 (s, 1H, Ar-CH), 3.19 and 3.41 (AB-q system, $J=20.5 \mathrm{~Hz}, 2 \mathrm{H}), 2.40(\mathrm{~s}, 2 \mathrm{H}), 2.22$ (s, $\left.3 \mathrm{H}, \mathrm{CH}_{3}\right), 1.07$ (s, 3H, $\left.\mathrm{CH}_{3}\right), 0.96\left(\mathrm{~s}, 3 \mathrm{H}, \mathrm{CH}_{3}\right),{ }^{13} \mathrm{C} \mathrm{NMR}$ $\left(\mathrm{CDCl}_{3}, 100 \mathrm{MHz}\right): \delta 196.5,162.1,158.3,142.7,141.1$, $136.3,135.7,132.3,128.8,128.2,126.7,115.5,64.5,50.7$, 40.8, 29.2, 27.3, 20.5. Anal. Calcd. for $\mathrm{C}_{24} \mathrm{H}_{22} \mathrm{~N}_{2} \mathrm{O}_{3}(\%)$ : C, 74.59; H, 5.74; N, 7.25; Found (\%): C, 74.50; H, 5.65; N, 7.12 .

(13) 2,3,4,13-Tetrahydro-3,3-dimethyl-13-(2-hydroxy-phenyl)-1 $H$-indazolo[1,2- $b]$ phthalazine-1,6,11-trione (4m). Yellow solid, mp. $184-188^{\circ} \mathrm{C}$. IR $\left(\mathrm{KBr}, v_{\max } \mathrm{cm}^{-1}\right) 2896,1662$, 1492, 1377, 1329, 1262, 1081, 791. ${ }^{1} \mathrm{H}$ NMR $\left(\mathrm{CDCl}_{3}, 400\right.$ MHz, TMS): $\delta 10.99$ (br s, $1 \mathrm{H}, \mathrm{OH}), 8.25-8.33(\mathrm{~m}, 2 \mathrm{H})$, 7.84-7.86 (m, 2H), 6.96-7.55 (m, 4H, Ar-H), $6.34(\mathrm{~s}, 1 \mathrm{H}$, Ar-CH), 2.43 and 2.60 (AB-q system, $J=17.3 \mathrm{~Hz}, 2 \mathrm{H}$ ), $2.32(\mathrm{~s}, 2 \mathrm{H}), 1.02\left(\mathrm{~s}, 3 \mathrm{H}, \mathrm{CH}_{3}\right), 0.96\left(\mathrm{~s}, 3 \mathrm{H}, \mathrm{CH}_{3}\right) ;{ }^{13} \mathrm{C} \mathrm{NMR}$ $\left(\mathrm{CDCl}_{3}, 100 \mathrm{MHz}\right): \delta 201.0,196.1,169.4,161.5,150.7$, $137.0,133.4,131.8,128.7,128.0,127.5,124.6,120.6$, 115.7, 111.0, 52.2, 43.2, 41.5, 27.2, 26.4. Anal. Calcd. for $\mathrm{C}_{23} \mathrm{H}_{20} \mathrm{~N}_{2} \mathrm{O}_{4}(\%)$ : C, 71.12; H, 5.12; N, 7.21; Found (\%): C, $70.98 ; \mathrm{H}, 5.03 ; \mathrm{N}, 7.08$.

(14) 2,3,4,13-Tetrahydro-3,3-dimethyl-13-benzo-[1,3]-dioxol -5-yl-1H-indazolo[1,2- $b]$ phthalazine-1,6,11-trione (4n). Yellow solid, mp. 194-198 ${ }^{\circ} \mathrm{C}$; IR $\left(\mathrm{KBr}, v_{\max } \mathrm{cm}^{-1}\right)$ : 2958 , $1662,1624,1489,1361,1261,825 .{ }^{1} \mathrm{H}$ NMR $\left(\mathrm{CDCl}_{3}, 400\right.$ MHz, TMS): $\delta$ 8.26-8.36 (m, 2H), 7.84-7.86 (m, 2H),
6.64-6.77 (m, 3H, Ar-H), 6.34 (s, 1H, Ar-CH), 5.92 (s, $\mathrm{CH}_{2}$-piperonyl), 3.20 and 3.42 (AB-q system, $J=19.8 \mathrm{~Hz}$, $2 \mathrm{H}), 2.43(\mathrm{~s}, 2 \mathrm{H}), 1.10\left(\mathrm{~s}, 3 \mathrm{H}, \mathrm{CH}_{3}\right), 1.01\left(\mathrm{~s}, 3 \mathrm{H}, \mathrm{CH}_{3}\right) ;{ }^{13} \mathrm{C}$ NMR $\left(\mathrm{CDCl}_{3}, 100 \mathrm{MHz}\right): \delta 195.5,162.1,155.7,147.7$, $145.9,138.3,134.6,133.2,129.8,127.8,121.5,118.8$, 115.6, 109.07, 100.7, 64.7, 50.7, 40.8, 29.2, 27.4. Anal. Calcd. for $\mathrm{C}_{24} \mathrm{H}_{20} \mathrm{~N}_{2} \mathrm{O}_{5}$ (\%): C, 69.22; H, 4.84; N, 6.73; Found (\%): C, 69.16; H, 4.73; N, 6.70.

(15) 2,3,4,13-Tetrahydro-3,3-dimethyl-13-(2-hydroxy-naphthalen-1-yl)-1 $H$-indazolo[1,2-b]phthalazine-1,6,11-trione (4o). Brown solid, mp. $156-160^{\circ} \mathrm{C}$; IR $\left(\mathrm{KBr}, v_{\max } \mathrm{cm}^{-1}\right)$ : 3155, 2958, 2927, 2855, 2253, 1718, 1628, 1466, 1376, 1293, 908, 734. ${ }^{1} \mathrm{H}$ NMR $\left(\mathrm{CDCl}_{3}, 400 \mathrm{MHz}, \mathrm{TMS}\right): \delta 10.72$ (br s, $1 \mathrm{H}, \mathrm{OH}), 8.23-8.30(\mathrm{~m}, 2 \mathrm{H}), 7.90-7.93(\mathrm{~m}, 2 \mathrm{H})$, 7.54-7.74 (m, 6H, Ar-H), 6.32 (s, 1H, Ar-CH), 3.48 and 3.61 (AB-q system, $J=17.6 \mathrm{~Hz}, 2 \mathrm{H}), 2.34(\mathrm{~s}, 2 \mathrm{H}), 1.12$ (s, $\left.3 \mathrm{H}, \mathrm{CH}_{3}\right), 1.01\left(\mathrm{~s}, 3 \mathrm{H}, \mathrm{CH}_{3}\right) ;{ }^{13} \mathrm{C} \mathrm{NMR}\left(\mathrm{CDCl}_{3}, 100 \mathrm{MHz}\right)$ : $\delta$ 193.2, 164.8, 160.2, 153.4, 139.1, 132.8, 132.4, 131.3, $129.4,129.1,128.5,127.7,125.2,124.4,122.9,119.1$, 118.5, 111.2, 49.6, 46.1, 43.1, 25.2, 22.7. Anal. Calcd. for $\mathrm{C}_{27} \mathrm{H}_{22} \mathrm{~N}_{2} \mathrm{O}_{4}(\%)$ : C, 73.96; H, 5.06; N, 6.39; Found (\%): C, $73.85 ; \mathrm{H}, 4.93 ; \mathrm{N}, 6.32$.

(16) 2,3,4,13-Tetrahydro-13-ethyl-1 $H$-indazolo[1,2-b]phthalazine-1,6,11-trione (4p). Yellow solid, mp. 164$166^{\circ} \mathrm{C}$. IR (KBr, $\left.v_{\max } \mathrm{cm}^{-1}\right)$ : 3461, 2877, 1731, 1652, 1456, 1372, 1291, 1176, 956, 892, 795, 701. ${ }^{1} \mathrm{H}$ NMR $\left(\mathrm{CDCl}_{3}\right.$, $400 \mathrm{MHz}, \mathrm{TMS}): \delta 8.28-8.32(\mathrm{~m}, 2 \mathrm{H}), 7.81-7.85(\mathrm{~m}, 2 \mathrm{H})$, $5.67(\mathrm{~m}, 1 \mathrm{H}, \mathrm{CHN}), 2.45-2.54\left(\mathrm{~m}, 2 \mathrm{H}, \mathrm{CH}_{2}\right), 2.27-2.33(\mathrm{~m}$, $\left.2 \mathrm{H}, \mathrm{CH}_{2}\right), 2.17-2.23\left(\mathrm{~m}, 2 \mathrm{H}, \mathrm{CH}_{2}\right), 1.96-2.06(\mathrm{~m}, 2 \mathrm{H}$, $\left.\mathrm{CH}_{2} \mathrm{CH}_{3}\right), 0.70$ (t, $\left.3 \mathrm{H}, \mathrm{CH}_{3}\right) ;{ }^{13} \mathrm{C} \mathrm{NMR}\left(\mathrm{CDCl}_{3}, 100 \mathrm{MHz}\right)$ : $\delta$ 197.3, 165.5, 162.2, 141.5, 134.4, 133.4, 128.9, 128.7, 127.8, 127.4, 117.6, 61.5, 37.0, 29.6, 22.3, 20.4, 9.9. Anal. Calcd. for $\mathrm{C}_{17} \mathrm{H}_{16} \mathrm{~N}_{2} \mathrm{O}_{3}(\%)$ : C, 68.91; H, 5.44; N, 9.45; Found (\%): C, 68.74; H, 5.27; N, 9.34.

(17) 2,3,4,13-Tetrahydro-13-propyl-1 $H$-indazolo[1,2-b]phthalazine-1,6,11-trione (4q). White solid, mp. 172$174^{\circ} \mathrm{C}$ [58]. IR (KBr, $\left.v_{\max } \mathrm{cm}^{-1}\right): 3165,2963,1662,1492$, 1377, 1262, 1081, 825, 791, 683. ${ }^{1} \mathrm{H}$ NMR $\left(\mathrm{CDCl}_{3}, 400\right.$ MHz, TMS): $\delta 8.07-8.22(\mathrm{~m}, 2 \mathrm{H}), 7.67-7.70(\mathrm{~m}, 2 \mathrm{H}), 4.91$ (m, 1H, CHN), 2.41-2.46 (m, 2H, $\left.\mathrm{CH}_{2}\right), 2.10-2.17(\mathrm{~m}, 2 \mathrm{H}$, $\left.\mathrm{CH}_{2}\right), 1.47-1.54$ (q, $\left.2 \mathrm{H}, \mathrm{CH}_{2} \mathrm{CH}_{2}\right), 1.31-1.43(\mathrm{~m}, 2 \mathrm{H}$, $\left.\mathrm{CH}_{2} \mathrm{CH}_{3}\right), 1.17-1.26\left(\mathrm{~m}, 2 \mathrm{H}, \mathrm{CH}_{2}\right), 0.76-0.83$ (t, 3H, $\mathrm{CH}_{3}$ ); ${ }^{13} \mathrm{C}$ NMR $\left(\mathrm{CDCl}_{3}, 100 \mathrm{MHz}\right): \delta 195.4,161.2,158.9,143.3$, 132.5, 132.3, 128.4, 121.3, 52.2, 41.3, 32.2, 29.7, 18.4, 18.2, 13.9. Anal. Calcd. for $\mathrm{C}_{18} \mathrm{H}_{18} \mathrm{~N}_{2} \mathrm{O}_{3}(\%)$ : C, 69.66; H, 5.85; N, 9.03; Found (\%): C, 69.53; H, 5.76; N, 8.94.

(18) 2,3,4,13-Tetrahydro-3,3-dimethyl-13-isobutyl- $1 H$ indazolo[1,2-b]phthalazine-1,6,11-trione (4r). Yellow solid, mp. 135-137 ${ }^{\circ} \mathrm{C}$ [57]. IR (KBr, $\left.v_{\max } \mathrm{cm}^{-1}\right): 3166,2896,1663$, 1492, 1377, 1262, 1082, 825, 791, 683. ${ }^{1} \mathrm{H}$ NMR $\left(\mathrm{CDCl}_{3}\right.$, $400 \mathrm{MHz}, \mathrm{TMS}): \delta 7.90-7.95(\mathrm{~m}, 2 \mathrm{H}), 7.51-7.56(\mathrm{~m}, 2 \mathrm{H})$, $4.98(\mathrm{~m}, 1 \mathrm{H}, \mathrm{CHN}), 3.26$ and 3.43 (AB-q system $J=20.52$ $\mathrm{Hz}, 2 \mathrm{H}), 2.33$ (s, 2H, $\left.\mathrm{CH}_{2}\right), 2.10-2.15\left(\mathrm{~m}, 2 \mathrm{H}, \mathrm{CH}-\mathrm{CH}_{2}\right.$ ), $1.87-1.92\left(\mathrm{~m}, 1 \mathrm{H}, \mathrm{CH}\left(\mathrm{CH}_{3}\right)_{2}\right), 1.10\left(\mathrm{~s}, 3 \mathrm{H}, \mathrm{CH}_{3}\right), 0.98(\mathrm{~s}$, $\left.3 \mathrm{H}, \mathrm{CH}_{3}\right), 0.83-0.85\left(\mathrm{~d}, 3 \mathrm{H}, \mathrm{CH}_{3}\right), 0.66-0.69\left(\mathrm{~d}, 3 \mathrm{H}, \mathrm{CH}_{3}\right)$; 
${ }^{13} \mathrm{C}$ NMR $\left(\mathrm{CDCl}_{3}, 100 \mathrm{MHz}\right): \delta 194.7,160.3,158.8,137.7$, 130.4, 129.2, 126.8, 119.3, 51.2, 38.3, 36.4, 31.4, 26.3, 24.2, 22.3, 20.1. Anal. Calcd. for $\mathrm{C}_{21} \mathrm{H}_{24} \mathrm{~N}_{2} \mathrm{O}_{3}(\%)$ : C, 71.57; $\mathrm{H}$, 6.86; N, 7.95; Found (\%): C, 71.44; H, 6.74; N, 7.80.

(19) 2,3,4,13-Tetrahydro-3,3-dimethyl-13-hexyl-1 $\mathrm{H}$ indazolo[1,2- $b]$ phthalazine-1,6,11-trione (4s). Yellow solid, mp. $84-86^{\circ} \mathrm{C}$ [57]. IR $\left(\mathrm{KBr}, v_{\max } \mathrm{cm}^{-1}\right): 2958,2926,1660$, 1632, 1462, 1375, 1288, 1180, 960, 892, 793, 699. ${ }^{1} \mathrm{H}$ NMR $\left(\mathrm{CDCl}_{3}, 400 \mathrm{MHz}, \mathrm{TMS}\right): \delta 8.24-8.34(\mathrm{~m}, 2 \mathrm{H}), 7.86-7.94$ $(\mathrm{m}, 2 \mathrm{H}), 5.67(\mathrm{~m}, 1 \mathrm{H}, \mathrm{CHN}), 3.18$ and 3.34 (AB-q system $J$ $=19.6 \mathrm{~Hz}, 2 \mathrm{H}), 2.47-2.51(\mathrm{~m}, 1 \mathrm{H}, \mathrm{CHN}), 2.40-2.44$ (dd, $\left.2 \mathrm{H}, \mathrm{CH}_{2} \mathrm{C}\right), 2.12-2.14(\mathrm{~m}, 1 \mathrm{H}, \mathrm{CH}), 1.15-1.33(\mathrm{~m}, 12 \mathrm{H}, 2$ $\mathrm{CH}_{3}$ and $\left.3 \mathrm{CH}_{2}\right), 1.10-1.14\left(\mathrm{~m}, 2 \mathrm{H}, \mathrm{CH}_{2}\right), 0.85-0.87(\mathrm{t}, 3 \mathrm{H}$, $\left.\mathrm{CH}_{3}\right) ;{ }^{13} \mathrm{C} \mathrm{NMR}\left(\mathrm{CDCl}_{3}, 100 \mathrm{MHz}\right): \delta 192.7,160.2,158.6$, $142.2,132.5,131.8,128.2,121.7,55.8,52.4,33.1,31.4$, 28.6, 27.0, 25.1, 23.5, 17.4, 14.5. Anal. Calcd. for $\mathrm{C}_{23} \mathrm{H}_{28} \mathrm{~N}_{2} \mathrm{O}_{3}(\%)$ : C, 72.60; H, 7.42; N, 7.36; Found (\%): C, $72.53 ; \mathrm{H}, 7.31 ; \mathrm{N}, 7.25$.

\section{Results and discussion}

Initially, equimolar amounts of phthalhydrazide $\mathbf{1}$, benzaldehyde 2a and cyclohexane-1,3-dione 3a were stirred at ambient temperature in ethanol. After $22 \mathrm{~h}$, yields of only $36 \%$ were obtained. To both improve the yield and optimize the reaction conditions, various Lewis acids were screened in a model reaction and the best yield was obtained using CAN as a catalyst (Scheme 1). The results are tabulated in Table 2.

A study was then done to determine the optimal loading of CAN. The reaction was carried out with varying amounts of catalyst, in the synthesis of $\mathbf{4 a}$. With 2 mol\% CAN, under similar conditions, a significant improvement was observed

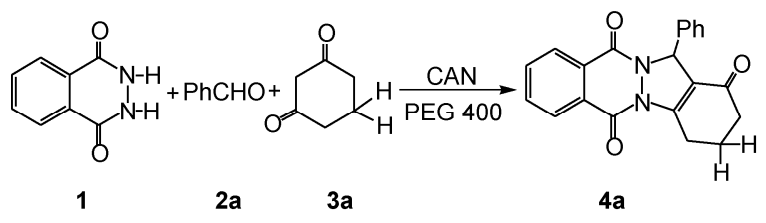

Scheme 1 Model reaction for the synthesis of $1 H$-indazolo[1,2- $b$ phthalazine-1,6,11-triones.

Table 2 Screening of various Lewis acids for the model reaction ${ }^{\text {a) }}$

\begin{tabular}{cccc}
\hline Entry & Catalyst & Time $(\mathrm{h})$ & Yield $(\%)^{\text {b) }}$ \\
\hline 1 & $\mathrm{InCl}_{3}$ & 12 & 62 \\
2 & $\mathrm{FeCl}_{3}$ & 8 & 46 \\
3 & $\mathrm{I}_{2}$ & 5.5 & 66 \\
4 & $\mathrm{CuSO}_{4}$ & 7 & 71 \\
5 & $\mathrm{CAN}$ & 2 & 94 \\
6 & $\mathrm{ZnCl}_{2}$ & 10 & 53 \\
7 & $\mathrm{AlCl}_{3}$ & 8.5 & 57 \\
\hline
\end{tabular}

a) Reaction conditions: phthalhydrazide $\mathbf{1}(1 \mathrm{mmol})$, benzaldehyde $\mathbf{2 a}$ (1 mmol), cyclohexane-1,3-dione 3a (1 mmol); catalyst: $5 \mathrm{~mol} \%$; solvent: PEG 400 (2 mL); $T: 50^{\circ} \mathrm{C}$. b) Isolated yields. and the yield of $\mathbf{4 a}$ was dramatically enhanced to $68 \%$ after stirring the mixture for $8 \mathrm{~h}$ (Table 3, Entry 2). Upon increasing the loading to $5 \mathrm{~mol} \%$, a decreased reaction time of only $2 \mathrm{~h}$ was required, and the product yield increased remarkably from $68 \%$ to $94 \%$ (Table 3, Entry 3). Although required reaction time remained the same upon using $10 \mathrm{~mol} \% \mathrm{CAN}$, the yield actually decreased from $94 \%$ to $76 \%$ (Table 3, Entry 4). A possible explanation for this lower product yield, is that the substrate (aldehyde) or product may be destroyed in the presence of excess amounts of CAN (10 mol\%). This shows that the catalyst concentration plays a major role in the optimization of the product yield.

The effect of temperature was also evaluated for the model reaction. The reaction proceeded poorly at room temperature. Elevating the reaction temperature proved helpful and the yield of the desired product $4 \mathbf{a}$ increased considerably. We were pleased to find that the reaction proceeded smoothly, and to almost complete conversion at $50^{\circ} \mathrm{C}$, affording $4 \mathrm{a}$ in $94 \%$ yield within $2 \mathrm{~h}$. Increasing the temperature beyond $50^{\circ} \mathrm{C}$, reduces the product yield due to the oxidation of the reactant (aldehyde) by CAN at high temperature. To assess the reaction efficiencies in different solvents, a variety of solvent systems were screened using the model reaction. The results are summarized in Figure 1. PEG 400 is the optimal solvent for this reaction giving excellent yields.

The optimum conditions for the model case were then applied to various aromatic and aliphatic aldehydes as

Table 3 Catalytic activity evaluation for the synthesis of $1 H$-indazolo $[1,2-b]$ phthalazine-1,6,11-triones ${ }^{\text {a) }}$

\begin{tabular}{cccc}
\hline Entry & CAN $(\mathrm{mol} \%)$ & Time $(\mathrm{h})$ & Yield $(\%)^{\mathrm{b})}$ \\
\hline 1 & 0 & 18 & 35 \\
2 & 2 & 8 & 68 \\
3 & 5 & 2 & 94 \\
4 & 10 & 2 & 76 \\
\hline
\end{tabular}

a) Reaction conditions: phthalhydrazide $\mathbf{1}(1 \mathrm{mmol})$, benzaldehyde $\mathbf{2 a}$ (1 mmol), cyclohexane-1,3-dione 3a (1 mmol); solvent: PEG 400 (2 mL); T: $50^{\circ} \mathrm{C}$. b) Isolated yields.

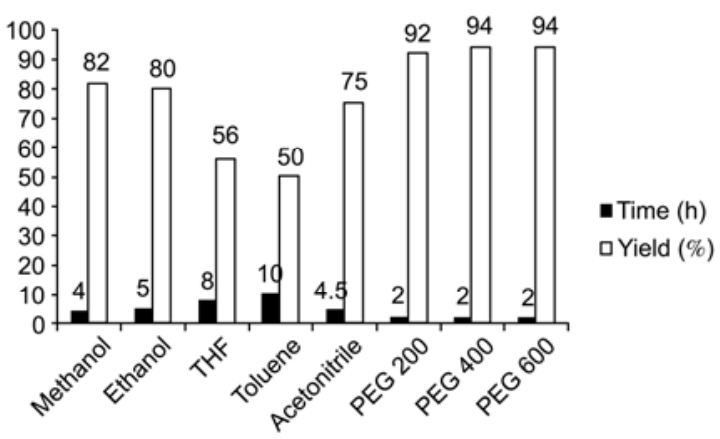

Figure 1 Effect of various solvents on the synthesis of $1 H$-indazolo [1,2-b]phthalazine-1,6,11-triones. Reaction conditions: phthalhydrazide $\mathbf{1}$ (1 mmol), benzaldehyde 2a (1 mmol), cyclohexane-1,3-dione 3a (1 mmol); catalyst: CAN (5 mol\%); solvent: x $(2 \mathrm{~mL}) ; T: 50^{\circ} \mathrm{C}$. Isolated yields. 
shown in Table 3. Aromatic aldehydes with both electron-donating and electron-withdrawing substituents proceeded smoothly to give excellent product yields. They also required reduced reaction times compared to aliphatic aldehydes, which reacted slowly to give only moderate yields (Scheme 2, Table 1, Entries 1-15). It is noteworthy that until now there has only been one report on the synthesis of $1 H$-indazolo[1,2- $b]$ phthalazine-1,6,11-triones using aliphatic aldehydes [57].

To establish the recyclability of the PEG 400, the reaction mixture was kept in a dry ice-acetone bath to precipitate the PEG 400. The desired product was extracted with solvent ether, in which the PEG 400 is immiscible. The remaining PEG 400 phase was reused in subsequent reactions. The reaction proceeded cleanly, exhibiting consistent results for up to four runs. A weight loss of approximately $5 \%$ was observed from cycle to cycle because of mechanical losses.

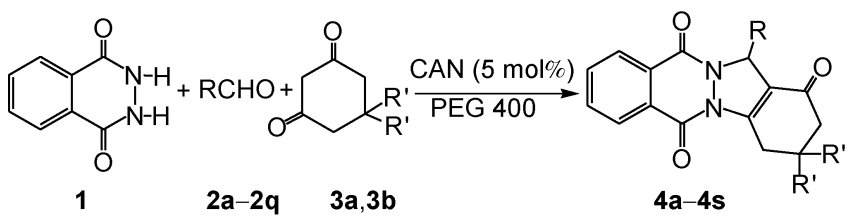

Scheme 2 CAN promoted synthesis of $1 H$-indazolo[1,2-b]phthalazine1,6,11-triones.

\section{Conclusion}

A novel and efficient catalytic method has been developed for the preparation of $1 H$-indazolo[1,2-b]phthalazine-1,6,11trione derivatives, using a ceric ammonium nitrate catalyst. The notable features of this clean one-pot procedure are the mild reaction conditions, improved yields, enhanced reaction rates, solvent recyclability and operational simplicity. This protocol represents a useful and attractive process for the synthesis of $1 H$-indazolo[1,2-b]phthalazine-1,6,11-trione derivatives.

Authors (Ritika Chauhan and Anwar Jahan) are thankful to University Grants Commission for the grant of Junior Research Fellowship. We also express our thanks to the Director of University Science and Instrumentation Centre, University of Delhi, Delhi for providing the spectral analysis data.

1 Zhu J, Bienayme H. Multicomponent Reactions. Wiley-VCH: Weinheim, 2005

2 Dömling A. Recent developments in isocyanide based multicomponent reactions in applied chemistry. Chem Rev, 2006, 106: 17-89

3 Ramón D J, Yus M. Asymmetric multicomponent reactions (AMCRs): The new frontier. Angew Chem Int Ed, 2005, 44: 1602 1634

4 Simon C, Constantieux T, Rodriguez J. Utilisation of 1,3-dicarbonyl derivatives in multicomponent reactions. Eur J Org Chem, 2004, 4957-4980

5 Orru R V A, de Greef M. Recent advances in solution-phase multicomponent methodology for the synthesis of heterocyclic compounds. Synthesis, 2003, 1471-1499
6 Bienayme H, Hulme C, Oddon G, et al. Maximizing synthetic efficiency: Multi-component transformations lead the way. Chem Eur J, 2000, 6: 3321-3329

7 Ulaczyk-Lesanko A, Hall D G. Wanted: New multicomponent reactions for generating libraries of polycyclic natural products. Curr Opin Chem Biol, 2005, 9: 266-276

8 Weber L. The application of multi-component reactions in drug discovery. Curr Med Chem, 2002, 9: 2085-2093

9 Hulme C, Gore V. Multi-component reactions: Emerging chemistry in drug discovery "from xylocain to crixivan". Curr Med Chem, 2003, 10: $51-80$

10 Dömling A, Ugi I. Multicomponent reactions with isocyanides. Angew Chem Int Ed, 2000, 39: 3168-3210

11 Kappe C O. Recent advances in the Biginelli dihydropyrimidine synthesis. New tricks from an old dog. Acc Chem Res, 2000, 33: 879-888

12 Franklin E C, Bergstrom F W. Heterocyclic nitrogen compounds PART I. Pentacyclic compounds. Chem Rev, 1935, 16: 305-361

13 Bergstrom F W. Heterocyclic nitrogen compounds. Part II A. Hexacyclic compounds: Pyridine, quinoline, and isoquinoline. Chem Rev, 1944, 35: 77-277

14 Lichtenthaler F W. Unsaturated $O$ - and $N$-heterocycles from carbohydrate feedstocks. Acc Chem Res, 2002, 35: 728-737

15 Litvinov V P. Multicomponent cascade heterocyclization as a promising route to targeted synthesis of polyfunctional pyridines. Russ Chem Rev, 2003, 72: 69-85

$16 \mathrm{Xu} \mathrm{Y,} \mathrm{Guo} \mathrm{Q} \mathrm{X.} \mathrm{Syntheses} \mathrm{of} \mathrm{heterocyclic} \mathrm{compounds} \mathrm{under} \mathrm{micro-}$ wave irradiation. Heterocycles, 2004, 63: 903-974

17 Vaughan W R. The chemistry of the phthalazines. Chem Rev, 1948, 43: 447-508

18 Clement R A. The oxidation of 2,3-dihydrophthalazine-1,4-dione with lead tetraacetate. Phthalazine-1,4-dione and 1,4-dihydropyridazino[1,2-b]-phthalazine-6,1l-dione. J Org Chem, 1960, 25: 17241727

19 Indelicato J M, Pasini C E. The acylating potential of $\gamma$-Lactam antibacterials: Base hydrolysis of bicyclic pyrazolidinones. J Med Chem, 1988, 31: 1227-1230

20 Kappe T, Kos C. A facile synthesis of 1-oxo- $1 H$-pyrazolo[1,2-a] pyrazol-4-ium-3-olates. Synthesis, 1989, 629-630

21 Turk C, Svete J, Stanovnik B, et al. Regioselective 1,3-dipolar cycloadditions of (1Z)-1-(Arylmethylidene)-5,5-dimethyl-3-oxopyrazolidin-1-ium-2-ide azomethine imines to acetylenic dipolarophiles. Helv Chim Acta, 2001, 84: 146-156

22 Clark M P, Laughlin S K, Laufersweiler M J, et al. Development of orally bioavailable bicyclic pyrazolones as inhibitors of tumor necrosis factor- $\alpha$ production. J Med Chem, 2004, 47: 2724-2727

23 Grasso S, De Sarro G, De Sarro A, et al. Synthesis and anticonvulsant activity of novel and potent 6,7-methylenedioxyphthalazin-1(2H)ones. J Med Chem, 2000, 43: 2851-2859

24 Nomoto Y, Obase H, Takai H, et al. Studies on cardiotonic agents. II. Synthesis of novel phthalazine and 1,2,3-benzotriazine derivatives. Chem Pharm Bull, 1990, 38: 2179-2183

25 Watanabe N, Kabasawa Y, Takase Y, et al. 4-Benzylamino1-chloro-6-substituted phthalazines: Synthesis and inhibitory activity toward phosphodiesterase 5. J Med Chem, 1998, 41: 3367-3372

26 Ryu C K, Park R E, Ma M Y, et al. Synthesis and antifungal activity of 6-arylamino-phthalazine-5,8-diones and 6,7-bis(arylthio)-phthalazine-5,8-diones. Bioorg Med Chem Lett, 2007, 17: 2577-2580

27 Li J, ZhaoY F, Yuan X Y, et al. Synthesis and anticancer activities of novel 1,4-disubstituted phthalazines. Molecules, 2006, 11: 574-582

28 Sinkkonen J, Ovcharenko V, Zelenin $\mathrm{K} \mathrm{N}$, et al. ${ }^{1} \mathrm{H}$ and ${ }^{13} \mathrm{C}$ NMR study of 1-hydrazino-2,3-dihydro- $1 H$-pyrazolo[1,2- $a$ ]pyridazine-5,8diones and $-1 H$-pyrazolo[1,2- $b]$ phthalazine-5,10-diones and their ring-chain tautomerism. Eur J Org Chem, 2002, 2046-2053

29 Mavel S, Thery I, Gueiffier A. Synthesis of imidazo[2,1-a] phthalazines, potential inhibitors of p38 MAP kinase. Prediction of binding affinities of protein ligands. Arch De Pharm, 2002, 335: 7-14

30 Carling R W, Moore K W, Street L J, et al. 3-Phenyl-6-(2-pyridyl) methyloxy-1,2,4-triazolo[3,4- $a$ ]phthalazines and Analogues: High- 
affinity $\gamma$-aminobutyric acid-A benzodiazepine receptor ligands with $\alpha 2, \alpha 3$, and $\alpha 5$-subtype binding selectivity over $\alpha 1$. J Med Chem, 2004, 47: 1807-1822

31 Street L J, Sternfeld F, Jelley R A, et al. Synthesis and biological evaluation of 3-heterocyclyl-7,8,9,10-tetrahydro (7,10-ethano)-1,2,4triazolo[3,4- $a]$ phthalazines and analogues as subtype-selective inverse agonists for the $\mathrm{GABA}_{\mathrm{A}} \alpha 5$. benzodiazepine binding site. J Med Chem, 2004, 47: 3642-3657

32 Imamura $\mathrm{Y}$, Noda A, Imamura $\mathrm{T}$, et al. A novel methylthio metabolite of s-triazolo[3,4-a]phthalazine, a lead compound for the development of antianxiety drugs, in rats. Life Sci, 2003, 74: 29-36

33 Kim J S, Lee H J, Suh M E, et al. Synthesis and cytotoxicity of 1-substituted 2-methyl-1H-imidazo[4,5-g]phthalazine-4,9-dione derivatives. Bioorg Med Chem, 2004, 12: 3683-3686

34 Lebsack A D, Gunzner J, Wang B, et al. Identification and synthesis of $[1,2,4]$ triazolo[3,4- $a]$ phthalazine derivatives as high-affinity ligands to the a $2 \delta-1$ subunit of voltage gated calcium channel. Bioorg Med Chem Lett, 2004, 14: 2463-2467

$35 \mathrm{Wu} \mathrm{H}$, Chen X M, Wan Y, et al. Synthesis and luminescence of 7-amino-2H-indazolo[2,1- $b]$ phthalazine-1,6,11(13H)-triones catalyzed by silica sulfuric acid. Lett Org Chem, 2009, 6: 219-223

36 Hwang J Y, Choi H S, Gong Y D. Solid-phase synthesis of $[1,2,4]$ triazolo[ $[3,4-a]$ phthalazine and tetrazolo[ $[5,1-a]$ phthalazine derivatives. Tetrahedron Lett, 2005, 46: 3107-3110

37 Shaterian H R, Ghashang M, Feyzi M, et al. Silica sulfuric acid as an efficient catalyst for the preparation of $2 H$-indazolo[2,1-b]phthalazine-triones. Appl Catal A, 2008, 345: 128-133

38 Wang H J, Zhang X N, Zhang Z H, et al. Highly efficient threecomponent synthesis of $1 \mathrm{H}$-indazolo[1,2-b]phthalazinetrione derivatives catalyzed by heteropolyacids. Monatsh Chem, 2010, 141: 425-430

39 Fazaeli R, Aliyan H, Fazaeli N, et al. Heteropoly acid in ionic liquid-An efficient catalyst for the preparation of $2 H$-indazolo [2,1-b]phthalazine-triones. Open Catal J, 2010, 3: 14-18

40 Mosaddegh E, Hassankhani A. A rapid, one-pot, four-component route to $2 \mathrm{H}$-indazolo[2,1- $b]$-phthalazine-triones. Tetrahedron Lett, 2011, 52: 488-490

41 Sayyafi M, Seyyedhamzeh M, Khavasi H R, et al. One-pot, threecomponent route to $2 \mathrm{H}$-indazolo[2,1-b]phthalazine-triones. Tetrahedron, 2008, 64: 2375-2378

42 Nair V, Deepthi A. Cerium (IV) ammonium nitrates: A versatile single-electron oxidant. Chem Rev, 2007, 107: 1862-1891

43 Nair V, Balagopal L, Rajan R, et al. Recent advances in synthetic transformations mediated by cerium(IV) ammonium nitrate. Acc Chem Res, 2004, 37: 21-30

44 More S V, Sastry M N V, Yao C F, et al. Cerium (IV) ammonium nitrate (CAN) as a catalyst in tap water: A simple, proficient and green approach for the synthesis of quinoxalines. Green Chem, 2006, 8: 91-95

45 Surendra K, Krishnaveni N S, Rao K R, et al. A mild and efficient procedure for the oxidation of epoxides and aziridines using
cerium(IV) ammonium nitrate and NBS. Tetrahedron Lett, 2005, 46: 4111-4113

46 Chen J, Spear S K, Huddleston J G, et al. Polyethylene glycol and solutions of polyethylene glycol as green reaction media. Green Chem, 2005, 7: 64-82

47 Zhang $\mathrm{Z} \mathrm{H}$, Yin $\mathrm{L}$, Wang $\mathrm{Y} \mathrm{M}$, et al. Indium tribromide in poly(ethylene glycol) (PEG): A novel and efficient recycle system for chemoselective deprotection of 1,1-diacetates. Green Chem, 2004, 6: 563-565

48 Kumar R, Chaudhary P, Nimesh S, et al. Polyethylene glycol as a non-ionic liquid solvent for michael addition reaction of amines to conjugated alkenes. Green Chem, 2006, 8: 356-358

49 Harris J M. Poly (Ethylene Glycol) Chemistry. In Biotechnological Applications. New York: Plenum Press, 1992. 3

50 Harris J M, Zalipsky S. Poly(Ethylene Glycol): Chemistry and Biological Applications (ACS Symposium). Washington DC: American Chemical Society, 1998

51 Mao J, Guo J, Fang F, et al. Highly efficient copper(0)-catalyzed Suzukie Miyaura cross-coupling reactions in reusable PEG-400. Tetrahedron, 2008, 64: 3905-3911

52 Mukhopadhyay C, Tapaswi P K. PEG-mediated catalyst-free expeditious synthesis of 2-substituted benzimidazoles and bis-benzimidazoles under solvent-less conditions. Tetrahedron Lett, 2008, 49: 6237-6240

53 Kouznetsov V V, Merchan Arenas D R, Romero Bohorquez A R. PEG-400 as green reaction medium for Lewis acid-promoted cycloaddition reactions with isoeugenol and anethole. Tetrahedron Lett, 2008, 49: 3097-3100

54 Kidwai M, Bhatnagar D. Ceric ammonium nitrate (CAN) catalyzed synthesis of $N$-substituted decahydroacridine-1,8-diones in PEG. Tetrahedron Lett, 2010, 51: 2700-2703

55 Kidwai M, Bhatnagar D, Chauhan R. Potassium carbonate-mediated green and efficient synthesis of imidazo[2,1-b]-1,3,4-thiadiazoles using PEG as solvent. J Heterocycl Chem, 2011, doi: 10.1002/jhet.1037

56 Kidwai M, Chauhan R, Bhatnagar D. Eco-friendly synthesis of 2-aminothiazoles using Nafion- $\mathrm{H}$ as a recyclable catalyst in PEG-water solvent system. J Sulf Chem, 2011, 32: 37-44

57 Ghorbani-Vaghei R, Karimi-Nami R, Toghraei-Semiromi Z, et al. One-pot synthesis of aliphatic and aromatic $2 H$-indazolo[2,1- $b$ ] phthalazine-triones catalyzed by $N$-halosulfonamides under solvent-free conditions. Tetrahedron, 2011, 67: 1930-1937

58 Sabitha G, Srinivas C, Raghavendar A, et al. Phosphomolybdic acid (PMA)- $-\mathrm{SiO}_{2}$ as a heterogeneous solid acid catalyst for the one-pot synthesis of $2 \mathrm{H}$-indazolo[ $[1,2-b]$ phthalazine-triones. Helv Chim Acta, 2010, 93: 1375-1380

59 Khurana J M, Magoo D. Efficient one-pot synthesis of $2 \mathrm{H}$ indazolo[2,1-b]phthalazine-triones by catalytic $\mathrm{H}_{2} \mathrm{SO}_{4}$ in waterethanol or ionic liquid. Tetrahedron Lett, 2009, 50: 7300-7303

60 Nagarapu L, Bantu R, Mereyala H B, et al. TMSCl-mediated one-pot, three-component synthesis of $2 \mathrm{H}$-indazolo[2,1-b]phthalazine-triones. J Heterocycl Chem, 2009, 46: 728-731

Open Access This article is distributed under the terms of the Creative Commons Attribution License which permits any use, distribution, and reproduction in any medium, provided the original author(s) and source are credited. 